\title{
Properties of a microjoule-class fiber oscillator mode- locked with a SESAM
}

\author{
C. Lecaplain ${ }^{1}$, B. Ortaç ${ }^{2}$, G. Machinet ${ }^{3}$, J. Boullet ${ }^{3}$, M. Baumgartl ${ }^{4}$, T. Schreiber ${ }^{5}$, E. Cormier ${ }^{3}$, A. Hideur ${ }^{1}$ \\ ${ }^{1}$ CORIA UMR 6614, Université de Rouen, Avenue de l'université BP 12, 76801 Saint Etienne du Rouvray, France \\ ${ }^{2} U N A M$-Institute of Materials Science and Nanotechnology, Bilkent University, 06800 Bilkent, Ankara, Turkey \\ ${ }^{3}$ Université de Bordeaux-CNRS-CEA, CELIA, 351 cours de la Libération F-33405 Talence, France \\ ${ }^{+}$Institute of Applied Physics, Friedrich Schiller University, Albert-Einstein-Strasse 15, D-07745 Jena, Germany \\ ${ }^{5}$ Fraunhofer Institute for Applied Optics and Precision Engineering, Albert-Einstein-Str. 7, D-07745 Jena, Germany \\ Tel.: +33232953 736, E-mail: caroline.lecaplain@coria.fr
}

Energy scaling of ultrafast $\mathrm{Yb}$-doped fiber oscillators has experienced rapid progress largely driven by many applications that require high average power femtosecond pulses. The fundamental challenge for ultrafast fiber lasers relies on the control of excessive nonlinearity, which limits pulse energy. The development of all-normal dispersion laser cavities based on large-mode-area photonic crystal fibers (PCFs) has enabled significant energy scaling [1-3]. In particular, up to microjoule energy levels have been achieved from rod-type fiber-based oscillators [2-3]. In such lasers, pulse shaping is dominated by the strength of the mode-locking mechanism which determines the pulse properties. In this contribution, we report the generation of high-energy subpicosecond pulses from a highly normal dispersion fiber laser featuring an Yb-doped rod-type PCF and a largemode-area PCF [Fig.1(a)]. Passive mode-locking is achieved using saturable absorber mirrors (SAMs). We study the influence of the SAM parameters on performances obtained in this new class of fiber oscillators. The structures exhibit $>20 \%$ modulation depths and 500 fs relaxation time with resonant and antiresonant designs. The antiresonant SAM structures ensure absorption bandwidths $>45 \mathrm{~nm}$ while the resonant structures exhibit $\sim 20 \mathrm{~nm}$ bandwidths. Stable mode locking with average powers as high as $15 \mathrm{~W}$ at $15 \mathrm{MHz}$ repetition rate, corresponding to microjoule energy level are obtained with all the structures. However, pulse properties and pulse shaping mechanism distinguish between resonant and antiresonant designs. Using a broadband antiresonant SAM leads to generation of highly-chirped pulses with 30 ps duration and $10 \mathrm{~nm}$ spectral width [Fig.1(b)]. The output pulses are extra-cavity dechirped down to $550 \mathrm{fs}$ duration. By increasing the strength of the mode-locking mechanism through the combination of the SAM with the NPE process, we obtain shorter pulses with slightly boarder spectra. Indeed, the output pulse duration is decreased from 30 ps to 13 ps by adjusting the wave-plates settings. The dechirped pulse duration is then shortened to $450 \mathrm{fs}$. We note that the current laser performances are limited to $\sim 1 \mu \mathrm{J}$ by the available pump power. Using a resonant SAM structure, the output pulse duration is decreased to $7 \mathrm{ps}$ [Fig.1(b)]. This pulse shortening results from the spectral filtering induced by the limited SAM bandwidth. All these results are in good agreement with numerical simulations which will be discussed in this communication.
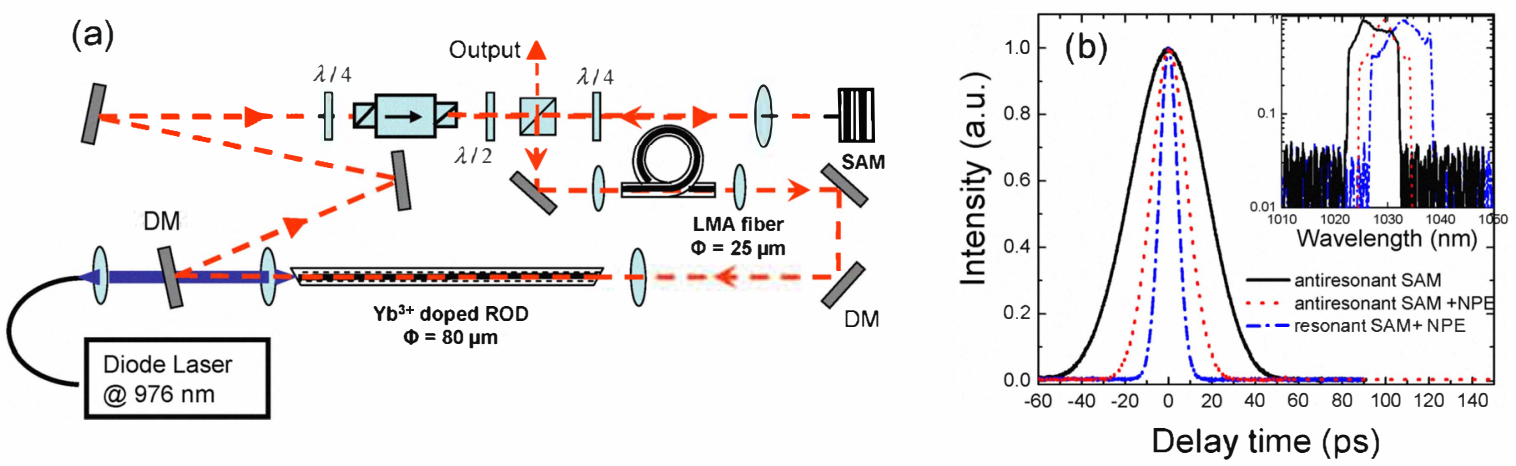

Fig. 1: (a) Setup of the photonic crystal rod laser. (b) Autocorrelation traces and optical spectra (inset) of the pulses obtained with antiresonant structure and resoant structures

\section{References}

[1] M. Baumgartl, B. Ortaç, C. Lecaplain, A. Hideur, J. Limpert and A. Tünnermann, "Sub-80 fs dissipative soliton large mode area fiber laser", Opt. Lett. 35, 2311-2313 (2010).

[2] B. Ortaç, M. Baumgartl, J. Limpert, and A. Tünnermann, "Approaching microjoule level pulse energy with mode-locked femtosecond fiber lasers," Opt. Lett. 34, 1585-1587 (2009).

[3] C. Lecaplain, B. Ortaç, G. Machinet, J. Boullet, M. Baumgartl, T. Schreiber, E. Cormier, and A. Hideur, "High-energy femtosecond photonic crystal fiber laser," Opt. Lett. 35, 3156-3158 (2010). 